\title{
Brain-Based Target Expansion
}

\author{
Daniel Afergan, Tomoki Shibata, Samuel W. Hincks, Evan M. Peck, Beste F. Yuksel, Remco Chang, \\ Robert J.K. Jacob \\ Tufts University \\ Medford, MA USA \\ \{afergan,tshibata,shincks,epeck02,byukse01,remco,jacob\}@cs.tufts.edu
}

\begin{abstract}
The bubble cursor is a promising cursor expansion technique, improving a user's movement time and accuracy in pointing tasks. We introduce a brain-based target expansion system, which improves the efficacy of bubble cursor by increasing the expansion of high importance targets at the optimal time based on brain measurements correlated to a particular type of multitasking. We demonstrate through controlled experiments that brain-based target expansion can deliver a graded and continuous level of assistance to a user according to their cognitive state, thereby improving task and speed-accuracy metrics, even without explicit visual changes to the system. Such an adaptation is ideal for use in complex systems to steer users toward higher priority goals during times of increased demand.
\end{abstract}

\section{Author Keywords}

brain-computer interface; BCI; fNIRS; adaptive interface; bubble cursor.

\section{ACM Classification Keywords}

H.5.m. Information Interfaces and Presentation (e.g. HCI): Miscellaneous

\section{INTRODUCTION}

In most graphical user interfaces (GUIs), navigating and pointing to an interface element is a necessary prerequisite to select it. A nearly incessant exchange between computer and user, clicking on graphical elements is a problem for which slight optimization can be extraordinarily impactful over longer sessions [6]. Consequently, considerable research has focused on improving users' speed and accuracy in basic point-and-click tasks [27, 50]. Small differences in interaction technique times can make much bigger differences in cognitive strategies and thus in high-level task performance beyond just the change in interaction time [18]. Because a system receives no extra information while a cursor travels through empty space [20], Grossman and Balakrishnan's bubble cursor creates an area cursor that always selects the closest target to the cursor [19]. Bubble cursor improves overall

Permission to make digital or hard copies of all or part of this work for personal or classroom use is granted without fee provided that copies are not made or distributed for profit or commercial advantage and that copies bear this notice and the full citation on the first page. Copyrights for components of this work owned by others than ACM must be honored. Abstracting with credit is permitted. To copy otherwise, or republish, to post on servers or to redistribute to lists, requires prior specific permission and/or a fee. Request permissions from permissions@ acm.org.

UIST 2014, October 5-8, 2014, Honolulu, HI, USA.

Copyright (C) 2014 ACM 978-1-4503-3069-5/14/10 ...\$15.00.

http://dx.doi.org/10.1145/2642918.2647414 performance compared to a standard cursor in these types of GUIs, and we introduce an augmentation which further enhances the technique's usability by integrating passive brain input as well as a ranking of the target's importance. While bubble cursor decreases movement time by always selecting the closest target, we can increase the effective width of highimportance targets with a variable parameter, making it easier to select these targets with no extra effort from the user. The variable parameter essentially gives us a flexible parameter that can be set in real time instead of defining it as a constant. Specifically, we propose a target expansion technique that aids the user in focusing on high importance objectives when necessary by monitoring the user's cognitive state and increasing the effective width of high-priority targets whenever we detect a state of multitasking.

In a pointing task, performance tends to suffer while the user is simultaneously completing multiple tasks with high cognitive workload [22]. It might be appropriate, therefore, to modify a user interface so that it facilitates the user's capacity to engage the essential on-screen elements. But the user's goals and capacity to meet those goals may change on a moment-to-moment basis, and an interface that emphasizes high-priority targets at all times comes at the expense of interaction with lower priority elements. Ideally, the interface should be sensitive to the user's context as it changes over time, dynamically and unobtrusively adapting to meet the user's needs. Unfortunately, standard cursor adaptations are limited to direct user input and by the transmission capacity between the user and the input device.

We propose to address this problem by incorporating physiological computing, which "has the potential to extend the communication bandwidth of HCI and enable a dynamic, individualised dialogue between user and system" [15] without any effort on the part of the user. By monitoring user cognitive state, we can tell when the user may be multitasking and aid the user in selecting targets of higher priority by increasing the width in bubble cursor calculations. Using this state information, we may be able to make higher priority targets easier to select at the expense of lower priority ones at critical moments, improving movement time and accuracy. In this paper, we propose a brain-computer interface (BCI) using functional near-infrared spectroscopy (fNIRS) as realtime input to influence target width in a pointing task.

In contrast to systems which use physiological data to determine when to turn on and off system automation, we use a more graded, continuous level of target adjustment, which 
aids the user without committing entirely. The system applies this assistance without any visual feedback to the user to avoid suggesting that the user has been deemed in need of help and spawning a feedback loop.

Small improvements to interface navigation systems can have a measurable impact on user performance in the long run [18]. These benefits may be particularly useful in high intensity interaction scenarios where a primary task demands the user's finite cognitive resources, leaving little for control of the interface. In some of these scenarios, the user must engage and hold in mind audio content while navigating a GUI. For example, an air traffic controller may be directing pilots while simultaneously interacting with graphical depictions of planes on the screen or an emergency response dispatcher may be communicating with units while selecting medical resources. Unmanned aerial vehicle operators speak to each other while monitoring vessels and financial traders may communicate while placing orders. These situations, which pose high and variable demands on the user's workload, call for seamless and adaptive interfaces. In performance-critical situations, we posit that an interface should sacrifice the ease of completing incidental goals in favor of more urgent goals.

We base our experiment on previous literature that shows the capability to detect cognitive multitasking signals in the anterior prefrontal cortex [29, 45, 46] using brain-sensing technologies and these patterns translate to dual verbal-spatial working memory tasks [33]. We use fNIRS, which shines near-infrared light at different wavelengths into the scalp and detects the amount of light that is reflected back. From this, we can infer changes in blood flow and oxygenated and deoxygenated hemoglobin levels in the prefrontal cortex, which correlate well to cognitive activities. The fNIRS sensors are comfortable, easy, and quick to setup, and they are nonintrusive so that a user can still perform tasks normally while wearing the sensor. Thus, it is ideal for passive physiological sensing because it can directly access brain signals without significantly disturbing the user, and it has been shown to measure working memory and multitasking [11, 29, 39]. In addition, recent research by McKendrick et al. [33] has shown that we can measure activation patterns during a dual verbal-spatial working memory task. Recent fNIRS literature has shown that we can continuously model fNIRS data and measure workload in real time [1, 23, 31, 38].

In this paper, we prototype and evaluate an area cursor system with brain-based dynamically expanding targets. To make the transition of expanding targets seem fluid, the user only witnesses the effect of the area cursor and does not see a visual increase in target size. In an interface with targets of variable importance, we increase the reach of bubble cursor to be more attracted to high-priority elements when we detect brain signals correlated to a cognitive multitasking signal (called branching). To demonstrate its efficacy and to configure adaptation and expansion parameters, we run a controlled experiment that modulates workload during a visual search and pointing task. We show that the time and accuracy savings by predicting user state correctly and helping the user easily navigate to high-priority targets outweighs the slight cost of the user having to move closer to a lower priority target when we guess incorrectly. In addition, we show that a constant expansion of high-priority targets actually leads to an overall decrease in performance, highlighting the importance of accurately and continuously measuring the user's need for this assistance and applying it only at the appropriate times.

This paper makes three primary contributions:

- We show that we can use fNIRS brain input to improve performance in a target selection task by dynamically updating a target's activation area.

- We demonstrate that adjusting the activation function, rather than the visual form of targets, can improve user performance without disrupting the user's mental model of the visual interface.

- We highlight a physiological interface that treats user input as a continuous measure, as opposed to binary or discrete user classification.

\section{BACKGROUND AND RELATED WORK}

Target acquisition tasks are often measured in terms of speed (movement time) and accuracy (error rate). We use the fundamental concepts of this field to design ways in which we can leverage human physical input with passive brain input.

\section{Movement Time and Bubble Cursor}

The general principle that accounts for the effectiveness of area cursors is a familiar and accepted one in HCI. Fitts' law measures the speed and accuracy of pointing performance, and as Kelso explains, "the relation between amplitude, movement time, and precision (or tolerance)" [28]. Although the formula has been altered for many purposes, a stable and widely used version is MacKenzie's 1992 formula, describing movement time (MT) as:

$$
M T=a+b \log _{2}\left(\frac{A}{W}+1\right)
$$

where $A$ is the amplitude (distance) of the movement and $W$ is the target width [30], $a$ and $b$ are interface constants, referring to the start/stop time of the device and device speed, respectively. The logarithmic portion of this equation is also known as the index of difficulty, and refers to the amount of work needed to reach a target. This implies that either increasing target size $(W)$ or decreasing distance $(A)$ on a pointing task will decrease movement time. Manipulating these variables allows the opportunity to improve performance in target selection tasks and lays the foundation for area cursors.

In 2005, Grossman and Balakrishnan first introduced the bubble cursor, a target acquisition technique that resized the activation area of a cursor to automatically select the closest target and provide the user visual feedback about the target selected [19]. In the Fitts' law equation, this mechanism increases effective target width, the size of a targets activation boundaries [19], and thus reduces movement time in a 
target acquisition scenario that includes surrounding distractor targets. Bubble cursor has shown consistently large performance improvements over a standard cursor, and custom tweaks can further enhance performance. Mott and Wobbrock's Bubble Lens augmentation of bubble cursor with an additional lens is the fastest pointing technique to date [36].

Other techniques focus on increasing target size by expanding clusters of targets [6], creating cascading menus with enlarged activation areas [9], or expanding targets once the cursor comes close [5]. These techniques expand the boundary of each target based on a Voronoi tessellation so that each target encompasses its maximum effective size without interfering with other targets. Zhai et. al showed that users performed better even when they did not anticipate an increase in target size [52]. Finally, McGuffin showed that pointing in these tasks is still dictated by Fitts' law and that "performance is governed by the target's final size, not its initial one" [32], even if the target only increases in size once the user has navigated the cursor close to the target. This indicates that users can take advantage of larger targets even if they were not expecting this increase.

While most of these variants have proven efficient in decreasing movement time by changing the width parameter in Fitts' law, they assume that the user is focused on the pointing task and do not consider situations where the user may be doing other work. In addition, these area cursors often entail an extra layer of visual distraction since they provide additional graphical feedback and stray from the traditional cursor model. Our goal is to add to the efficiency of the bubble cursor by subtly and dynamically adapting the size of the high-priority targets based on the user's cognitive state. In our custom version, we change the motor space of the uppercase targets, having the width increase and take up part of the motor space for lowercase letters (without visual indication).

\section{Brain-Computer Interfaces}

Since optimal target width may vary depending on the user's context or cognitive state, we monitor relevant cues in the user's changing physiology. We build off the field of passive brain-computer interfaces - systems that use input from brain sensors to covertly adapt their function, controls, or display automatically in response to changes in the users state. Passive systems stand in contrast to active BCIs, where neural correlates of the user's intention map directly to system inputs [51]. In target-selection-based active BCIs, the system responds explicitly based on user thought, directing the cursor and clicking by monitoring the brain's unique response when it imagines mouse navigation movements and activating a target $[14,43]$. While these projects are invaluable for people with limited motor function, the usability of brain-controlled navigation systems pales in comparison to an ordinary mouse. Instead, using passive BCI, our goal is to identify and leverage naturally-occurring user states to subtly enhance interaction target selection within the general population.

Cutrell and Tan suggest that the implicit commands inferred from a users changing brain activity may be the most promising, universal application of BCI [12]. The model must be very cautious since users are often in a different mental state during offline calibration and online feedback [47]. Researchers have recently begun exploring new types of passive, realtime BCI systems using fNIRS. Girouard et. al built an application which altered music based on task predictions from an fNIRS processing algorithm [17]. Solovey et al.'s Brainput system adapted in real-time to a scenario where an interactive human-robot system changed its state of autonomy based on whether it detected a particular state of multitasking [46] and Peck et al. demonstrated a passive adaptive movie recommendation system [37]. In these systems, the brain processing algorithms typically predict the user's mental activity on a discrete, often binary scale (e.g., high vs. low workload), which then maps one-to-one with a system adaptation. In the present experiment, we explore a more continuously adaptive scheme, where measures of classification confidence provide a proxy for differentiating the degree of multitasking.

\section{Brain Sensing with fNIRS}

Functional near-infrared spectroscopy (fNIRS) is an optical brain sensing technique that observes similar physiological parameters to functional magnetic resonance imaging (fMRI). Near-infrared light is sent into the forehead at frequencies of $690 \mathrm{~nm}$ and $830 \mathrm{~nm}$ which enters the tissue at depths of $1-3 \mathrm{~cm}$ [48]. Critically, light penetrates biological tissue and bone at these wavelengths but is absorbed by oxygenated (oxy-Hb) and deoxygenated hemoglobin (deoxy-Hb) in the bloodstream [26]. Since active neurons summon and consume oxygen, a sharp increase of blood flow [13] coupled with decreased levels of deoxy-Hb and increased levels of oxy-Hb tends to signify that a neural region at large is at work performing meaningful computation. Because the optical properties of deoxy-Hb and oxy-Hb differ, the attenuation of the near-infrared light at different wavelengths returning to the sensors on the forehead describes localized brain activation.

FNIRS signals have shown resliency against head movement, facial movement, ambient noise, heartbeat, and muscle movement [44]. Relying on simple and cheap fundamental technology, fNIRS is safe, comfortable, easy-to-setup, and has the potential for portability. The data from the device has been used to differentiate between levels of workload [3, 25, $37,42]$, verbal and spatial working memory [24], game difficulty levels [16], and cognitive multitasking. Putting this data to practical use, predictive models have been applied in real time to adapt user interfaces to these physiological states [45, 46].

\section{Multitasking and Cognitive Workload}

Working memory has been defined as the "temporary storage and manipulation of the information necessary for such complex tasks such as language comprehension, learning and reasoning" [4]. Repovš and Baddeley's [41] multicomponent model and Wickens' four-dimensional multiple resource model [49] both regard working memory as a limited pool of cognitive resources. Even if two different tasks, such as auditory and visual, do not interfere with each other, they are still competing for common resources. There is no perfect allocation of resources, and a strain on the auditory 
components of working memory affect the resources available to visual and motor components, and vice versa.

Specifically, visual search is not efficient while humans are manipulating information held in working memory. This was demonstrated in an experiment by Han and Kim [22] in which participants performed a visual search task while simultaneously performing other tasks (counting backwards from a digit or reordering alphabet letters). These conditions reduced speeds in the visual search task when compared to the searchalone condition [22]. The authors concluded that the extra load of the additional tasks affected the working memory's executive processes and hindered performance in a branching scenario.

Speed and error rate suffer when the amount of effort available for a point-and-click task is limited. Balancing accuracy and speed, the user's "two concurrent minimization efforts draw from a common pool of resources, and this pool is limited" [21]. In general, multitasking refers to the act of performing multiple tasks simultaneously in pursuit of various independent goals. This planning strategy requires cognitive resources located in different parts of the prefrontal cortex [7].

However, certain types of multitasking have different neural signatures and their activations can be distinguished. Koechlin et al. showed that although keeping a goal in mind over time or alternating between goals did not lead to an increased activation in the prefrontal cortex, this area of the brain is responsible for the "ability to hold in mind goals while exploring and processing secondary goals." [29] Koechlin et al. [29] describe a particular state of multitasking, branching, where the user must remember one or more primary goals, while simultaneously processing and shifting between secondary goals. In Koechlin's study, the participants' fMRI data exhibited significant activation in the anterior prefrontal cortex when the user was branching compared to other states of multitasking [29], and Solovey et al. demonstrated that this branching signal is distinguishable via fNIRS [45] and can be used to power an adaptive real-time system [46].

Because branching indicates that the user is focused on a primary task while working on a secondary goal, it is the ideal opportunity to adapt a system to guide the user's focus toward the essential parts of a secondary goal and reduce the total working memory load.

Based on the working memory and search task literature, we explore if we can aid the user in working memory tasks by adapting interaction with visual elements in a secondary task. When the user enters a state of branching, they will not be as efficient on the visual search task. In this circumstance, it benefits the user to focus attention on the main elements of the visual search. Therefore, when the fNIRS device detects evidence of this neurophysiological signal, the GUI should aid the user and focus on higher priority targets, increasing their effective width at the expense of the lower priority targets.

\section{FNIRS AS AN INPUT FOR MULTIMODAL TASK ADAPTA- TIONS}

In this paper, we explore the possibility of using fNIRS to provide useful changes to the backend of a visual interface. The main questions we sought to answer are:

- Can we use brain input to detect when a user is multitasking in a challenging multimodal task?

- Can we improve user performance in an on-screen task by using brain state without changing the visual interface?

\section{Adaptation Mechanism: Target Expansion}

We propose dynamically manipulating the size of highpriority targets as a function of the user's current state. During periods of difficult multitasking, the target size of highpriority targets expand, making them easier to click and the lower priority targets, in effect, comparatively evasive. Under less mentally strenuous circumstances, the size of highpriority targets returns to default settings. This manipulation allows the user to focus on high-priority targets during critical moments, without forfeiting access to low-priority targets during less demanding periods of work.

We introduce two important design features of adaptive target expansion. First, unlike traditional bubble cursors, as targets change in size, there are no changes to the visual representation of the targets. We make this design decision based on long-standing research on the sensitivity of our perceptual system to movement in the visual field. Frequent, unpredictable changes to the visual representation of the target could disrupt the user and renders the system more sensitive to inaccurate user classifications.

Second, we use a continuous adaptation mechanism. Rather than modifying target size between two system states (default target size and maximum target size) that directly correspond with two user states, the target size may also be anywhere between the default and maximum size, depending on output from the user model. We believe that this design creates a gentle, less obtrusive adaptation that is (again) less sensitive to potential misclassifications of the user model. We discuss the mapping of user state to target size in more detail in our system description.

\section{EXPERIMENT SCENARIO}

In this section, we explain how we control our adaptive simulation with brain-sensing, and provide evidence of its efficacy with a user study. Using fNIRS while the user is performing a calibration task, we build a machine learning model of what the user's brain activation looks like during a particular multitasking state, contrasting it against different forms of multitasking. When we detect branching multitasking during the simultaneous tasks, we modify the bubble cursor to aid the user in focusing on high-priority targets.

In order to test the efficacy of a system that dynamically adjusts target size based on passive brain input, we designed a highly-controlled multitasking scenario that involves both audio and visual interface tasks. The user's goal is to maximize performance in the primary task while also completing 

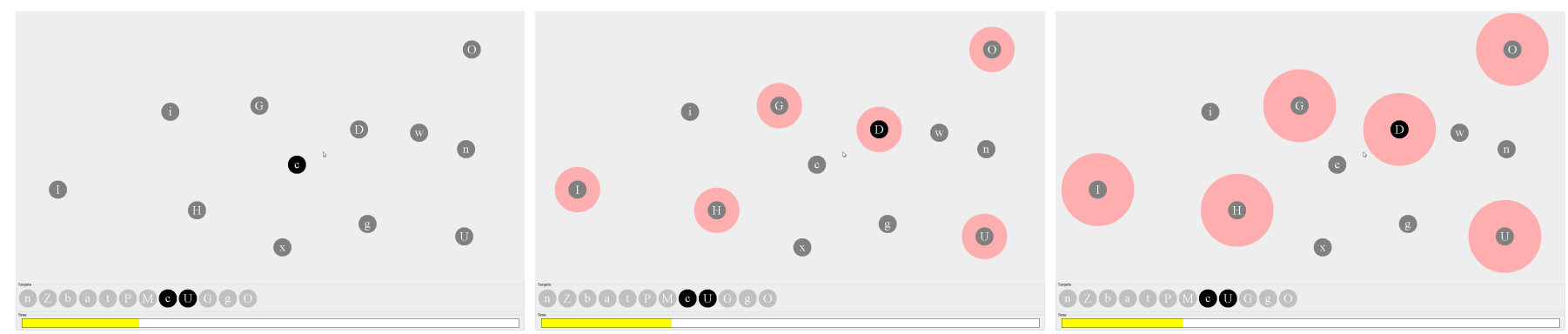

Figure 1. Simulated screen captures of our three target expansion conditions, with visual indications of expanded target widths. The letters on the bottom are the list of letters for the user to select, while the top is the visual search canvas. Although users never saw the expanded widths (indicated by light pink outer circles), these increased widths were used to make it easier for users to select the high-priority targets in the adaptive and static conditions. With no expansion(left), all targets have the same width. Although the users did not see these changes, we show the effects of target expansion in the adaptive expansion condition (center), where uppercase target expansion varied according to brain measures, and static expansion (right), where uppercase target widths were expanded by $300 \%$.

as much of the secondary task as possible. Because there is a performance tradeoff, we developed an adaptation with the goal of helping the user.

The users complete a known multitasking task, the "tablet" task, and we use a machine learning model of their cognitive activity during this task to predict their cognitive state and adapt the cursor while simultaneously performing n-back and visual search tasks.

\section{Primary Audio Task: N-Back}

In order to test our system, we chose a highly controlled task that is grounded in the psychology literature for our experimental testbed. We believe this work generalizes to realworld audio tasks that people typically engage in - communicating to pilots, emergency dispatchers talking to callers, and financial traders communicating during transactions.

A well-validated method to manipulate workload [34, 35, 40], the $n$-back task served as the primary task in this experiment. Participants performed an audio recall n-back task, which has been shown in multiple studies to increase task demand on a user as $n$ increases "without requiring direct conflict with manual control or visual processing demands" [35]. In the audio n-back, participants listened to a list of single-digit numbers and after each number, repeated out loud the number that they just heard (0-back), the previous number (1-back), or the number that they heard two numbers ago (2-back). Each nback set consisted of 16 individual trials, with each digit (0-9) being represented as one of the first ten digits, followed by a sequence of six non-repeating digits.

\section{Secondary Interface Task: Visual Search}

In order to construct a controlled task that mimics timesensitive interface selection activity, we designed an experimental secondary task based on visual search. Visual search tasks require a combination of visual and motor skills in order for users to find and select targets. In our task, users were presented with a canvas containing both uppercase and lowercase letters scattered randomly across the screen, as seen in Figure 1. At the bottom of the screen was a list of 12 letters (6 uppercase and 6 lowercase) as well as a timer.
Participants were instructed to click on letters from the list in sequential order. We consider the uppercase letters to be high-priority targets that may be modified given our adaptive strategy and the lowercase letters as low-priority targets. We show Figure 1 as a demonstration of the target expansions, but there was no visual indication of these changes. The width of the lowercase letters was never explicitly affected in any of the conditions, but occasionally decreased if they were bordered by expanded uppercase targets. Participants were informed that they could skip lowercase letters without penalty, but that they must click on all uppercase letters. However, they could not go back once they advanced in the list and skipped letters.

\section{Scoring}

Participants performed this visual search task concurrently with the audio n-back, and were instructed that n-back responses were worth 20 points, uppercase letters were worth ten points, and lowercase letters were worth five points. Their goal was to earn as many points as possible in the time allowed. They were told to go as quickly and accurately as possible and to click once the desired letter was highlighted. After each click on a correct target, the canvas reset and shuffled the locations of all the letters.

\section{SYSTEM DESIGN}

The user performs the simultaneous audio n-back and visual search task. During this, we use machine learning to predict the user's multitasking state. At each screen refresh (once the user selects a target), we use the confidence value of the model for the last five seconds as an expansion coefficient to increase the effective width of the uppercase letter targets. We compare the performance in this condition to two other controls: never expanding the target, and always completely expanding the target.

\section{Equipment}

We used a multichannel frequency domain Imagent fNIRS device from ISS Inc. (Champaign, IL) for data acquisition. Two probes were placed on each participant's forehead, as shown in Figure 2. Each probe had four light sources, each emitting light at $690 \mathrm{~nm}$ and $830 \mathrm{~nm}$, and one detector. The 


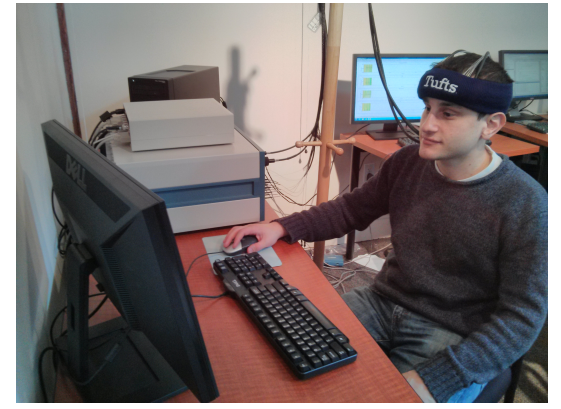

Figure 2. A user with two fNIRS probes held on with a headband.

source-detector distances ranged from 1.5 to $3.5 \mathrm{~cm}$ and the sensor sampling rate was $11.79 \mathrm{~Hz}$.

\section{Multitasking Calibration Task}

In order to induce branching in our subjects, we replicated Koechlin et al.'s multitasking experiment, which has been shown to produce neurophysiological changes during the branching condition measurable by both fMRI and fNIRS [29, 45, 46]. Participants were shown a series of uppercase and lowercase letters from the word "TABLET" and had to respond according to different rules for the delay, dualtask, and branching conditions.
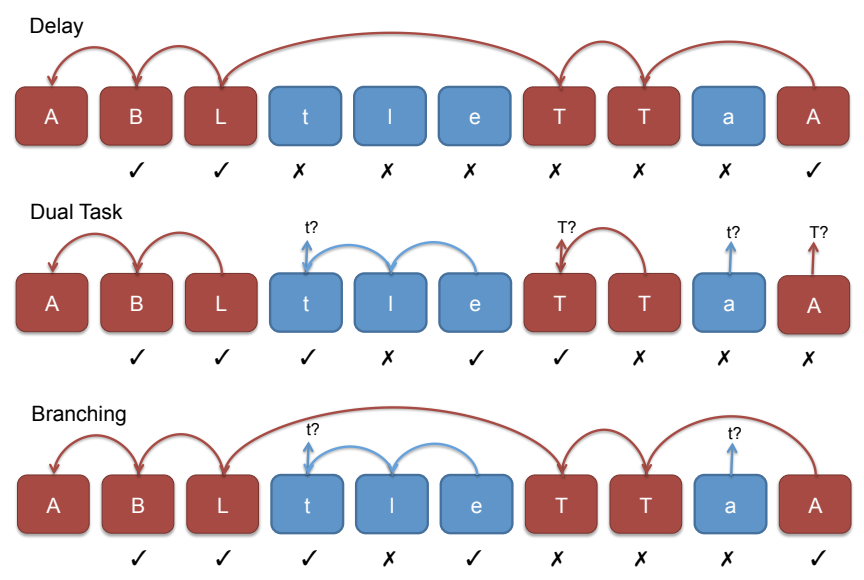

Figure 3. Conditions of the tablet task. Stimuli were uppercase (red) or lowercase (blue) letters, and participants responded according to rules for each task.

- In the Delay condition, the user must ignore the stimuli of a secondary task that is interrupting a primary task. Participants ignored lowercase letters and responded if two consecutively presented uppercase stimuli were in succession in the word "tablet."

- In the Dual-task condition, the user switches tasks without retaining any information about the previous task. Participants had to respond if two consecutive stimuli of the same case were in immediate succession in the word "tablet." However, if the two stimuli were of different cases, participants had to respond if the second stimulus was a ' $\mathrm{T}$ ' or 't.'

- In the Branching condition, participants had to keep information about one goal in mind while working on another.
Participants had to respond to the uppercase letters the same as the delay condition, always remembering the last uppercase letter. For lowercase letters, they responded the same as the dual-task condition, answering if the two consecutive stimuli were in immediate succession in "tablet" if they were both lowercase, but responding if stimulus was a ' $t$ ' for a change of case.

For each trial of the "tablet" task, participants viewed 11 letter stimuli, each presented for $500 \mathrm{~ms}$, with a $2500 \mathrm{~ms}$ period to respond between stimuli. Participants completed 32 trials of the "tablet" task, 16 branching and eight of both the delay and dual-task in order to ensure that the machine learning model had the same number of branching and non-branching trials. We kept the timing of stimuli and probability of a match identical to Koechlin et al., and ran the trials in random order. Although each trial was 33 seconds, we classified brain data on the first 15 seconds of brain data. A pilot study $(n=8)$ using the same task suggested that we could achieve similar or better accuracy using a short period for classification. There was a 15 second rest period between trials in order to allow the brain to return to its resting state, and participants were allowed to take a break after every ten trials. Although each participant had an individual model, the overall trends are shown in Figure 4. Our data logs, as well as Koechlin and Solovey's studies [29, 45], show strong similarities between the performance metrics for branching and dual-task conditions (while the control task is much easier), but the brain activation patterns for the branching task are differentiable from the dualtask and control task (which are similar). Therefore, for our machine learning model, we distinguish branching from nonbranching signals (delay + dual-task).

\section{FNIRS Signal}

During the tablet task, raw fNIRS data was collected by Boxy (software from ISS, Inc.) and sent to a specialized analysis system which was built in-lab using MATLAB. There, the system calculated the time series of change in fNIRS data over individual trials for each information channel (2 probes $\mathrm{x} 4$ light sources $\mathrm{x} 2$ wavelengths), labeling them as examples of either branching or non-branching. The signals were filtered for heart rate, respiration, and movement artifacts using a third-degree polynomial filter and low-pass elliptical filter. In the past, this process has been used to support other realtime fNIRS adaptive systems [1, 2, 37, 46].

\section{Machine Learning Model}

We constructed a model for the user multitasking states branching vs. non-branching (delay and dual-task), as Solovey et al. 2012 had also done. In order to determine user state, we built a machine learning model based on prefrontal cortex activation signals during the calibration task.

For each of the 32 "tablet" task trials, we calculated the mean and slope of the change in the light intensities for each of the 8 source-detector pairs at two different wavelengths. Thus, each trial consisted of 32 features.

We labeled each of these examples as branching or nonbranching and fed them to LIBSVM, a support vector machine classification system [8]. Per the recommendation of 
Cui et al. [10], we used a linear kernel to prevent overfitting the data and did a parameter search to find the best combination of $\mathrm{C}$ and $\gamma$ parameters for each user.

We used ten-fold cross-validation to ensure that the model was accurate, and we did not use data from two participants that could not be accurately modeled (cross-validation accuracy of less than $50 \%$ ).

For testing, the LIBSVM model received an input example and provided both a classification and a confidence measure (probability estimate) between $50 \%$ and $100 \%$. This allows us to receive predictions of intermediate states instead of just $0 \%$ and $100 \%$.

\section{Change in Oxy-Hb during Branching vs. Non-Branching}

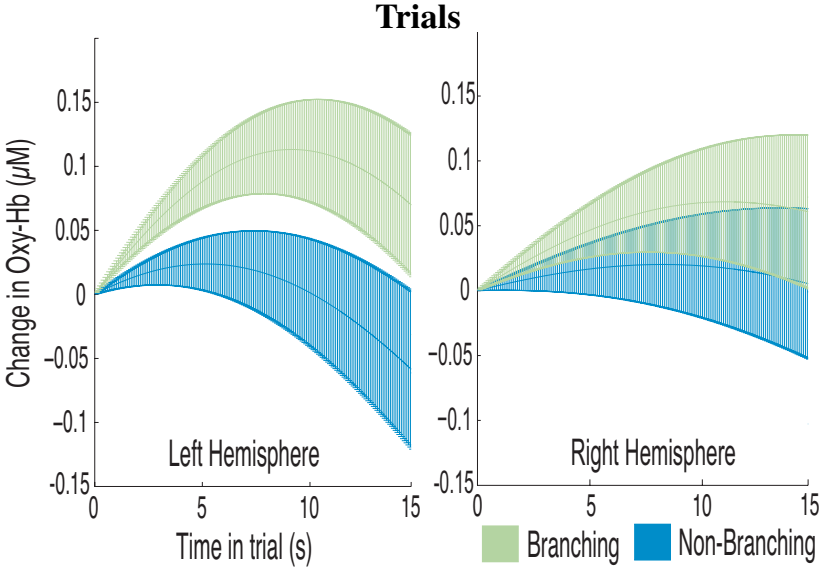

Figure 4. Although we model each participant individually, the fNIRS signal exhibited general trends as participants interacted with the branching and non-branching task. Plotting the mean change in oxy-Hb across all training trials and all participants (16 trials $* 12$ participants) shows that higher levels oxy-Hb correlate with the branching task. The standard error of the mean is shown with the width of the plots.

\section{Adaptation Trigger and Design}

The machine learning classifier provided a prediction every .5 seconds based on the last 15 seconds. We merged predictions from an overlapping sliding window of five seconds (with 4.5 seconds of overlap), computing the average certainty levels of the branching state. This means that the system used the average of the last ten classifications, discarding the oldest classification every time it received a new one. This measure directly controlled the uppercase letter target expansion: from no expansion up to three times the original target radius. The expansion radius changed only after each user click to avoid inconsistent cursor behavior.

\section{EXPERIMENT DESIGN AND PROCEDURE}

We ran 12 participants (five female) between the ages of 19 and 31. All participants were right-handed, native English speakers with no history of brain damage or disease and had normal or corrected-to-normal vision. Participants were paid for their time and incentivized with a performance bonus for their overall score. All participants were trained to proficiency on the tasks before testing.

Participants were tasked with simultaneously completing audio n-back and visual search tasks. Because of incentives in the point system, they considered the audio n-back their primary task and the visual search task their secondary task. We expected that the completely expanded targets would aid them to click during times of multitasking but would hinder the user in clicking in other situations.

Although we manipulated n-back difficulty level, the primary independent variable under investigation was the level of adaptation of system:

- no expansion: we use a standard area bubble cursor and no expansion of the uppercase letters. The cursor always selects the nearest target.

- static expansion: we expand the uppercase (priority) targets by three times the original radius and fix its size for the entire trial.

- adaptive expansion: the size of the uppercase targets increases and decreases as a function of the classifier's confidence that the user was in a branching state.

We expect that a brain-based target expansion will aid the user in clicking uppercase letters compared to the control condition of no adaptation, but the target expansion techniques leave less activation area for the lowercase letters, so the mean time to select lowercase letters will increase. We expect that these results will be extreme for the static condition, where users will be able to select uppercase targets the most quickly, but take the most time to select lowercase letters.

We also modified the difficulty of the primary task (the audio n-back) between 0-back (easy), 1-back (medium), and 2back (hard). Thus for each adaptive condition, participants simultaneously performed nine consecutive audio n-back trials (three 0-back, 1-back, and 2-back trials) and fourteen visual search trials. Each audio n-back lasted 40 seconds with a three second break, totaling 6 minutes and 27 seconds. Participants were given 25 seconds for each visual search task with a three second break, totaling 6 minutes and 32 seconds. Participants were told that the mouse cursor might sometimes be more attracted to uppercase letters in order to help them focus on the higher priority visual targets.

Before experimentation, a set of stimuli for the audio n-back and visual search tasks were randomly generated. This set was used for each participant and the order that tasks were presented was counterbalanced. In addition, the visual search tasks were designed so that there would be no overlap between targets, regardless of expansion.

\section{Dependent Measures}

In order to evaluate the interaction between adaptive techniques and multitasking condition, we recorded the following dependent measures:

- $N$-back accuracy rate: the percentage of correct answers in the primary audio n-back task. Participants gave 135 $\mathrm{n}$-back responses per condition.

- Number of targets selected: the average number of targets that the user selected, out of 168 targets (84 uppercase, 84 lowercase). 


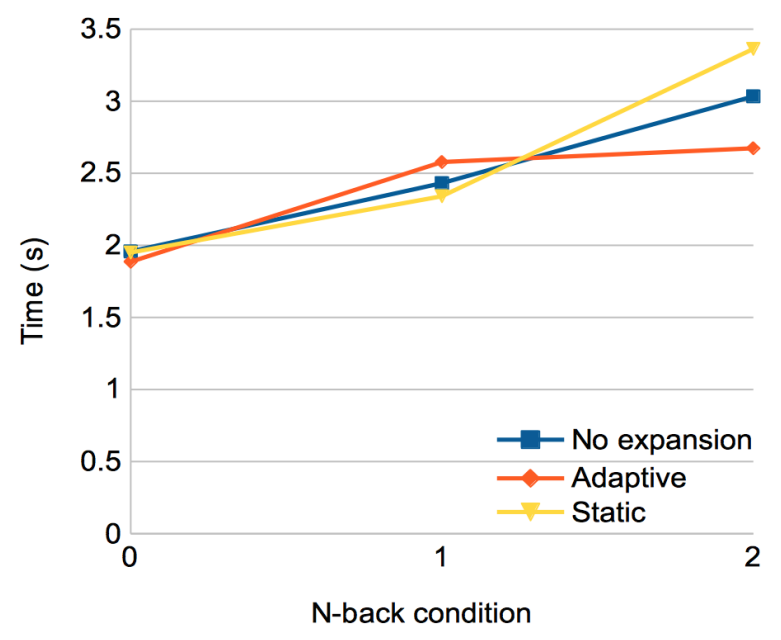

Figure 5. Movement time for uppercase letters by expansion condition.

- Combined score: the average score the user achieved, as measured with the weighted points system. The maximum total score was 3960 points.

- Movement Time: the average amount of time it took between target selection.

- Target acquisition error rate: the percentage of clicks that missed the target.

We hypothesize that if we can improve performance on the visual task by improving target selection and focusing on only high-priority targets when the user is busy, the audio n-back accuracy rate should remain constant or improve since the user will have extra cognitive resources.

\section{RESULTS}

\section{Task Performance Results}

A Kolmogorov-Smirnov test showed that there was a normal distribution of the data. We found that users performed better on both tasks with the adaptive condition compared to the other two conditions. With a two-way ANOVA, there were significant main effects of target expansion condition, $F(2,10)=4.24, p<.05$, and n-back condition, $F(2,10)=$ $49.14, p<0.001$ on the total number of correct n-back responses. A Tukey's pairwise comparison revealed that the adaptive condition significantly outperformed the static condition $(p<.001)$. We also found significant main effects of target condition, $F(2,10)=5.62, p=0.001$ and n-back condition, $F(2,10)=165, p<0.01$ on the total number of correct target clicks, and a Tukey's pairwise comparison analysis showed that the adaptive condition significantly outperformed both no expansion $(p<.05)$ and static $(p<.05)$. This also yielded a significant total difference on total score $(p<.005)$, with adaptive again outperforming no expansion $(p<.05)$ and static $(p<.05)$ conditions.

Users performed better on both tasks when we adaptively throttled the expansion of targets, but actually performed worst when they received the expansion for the entire trial.

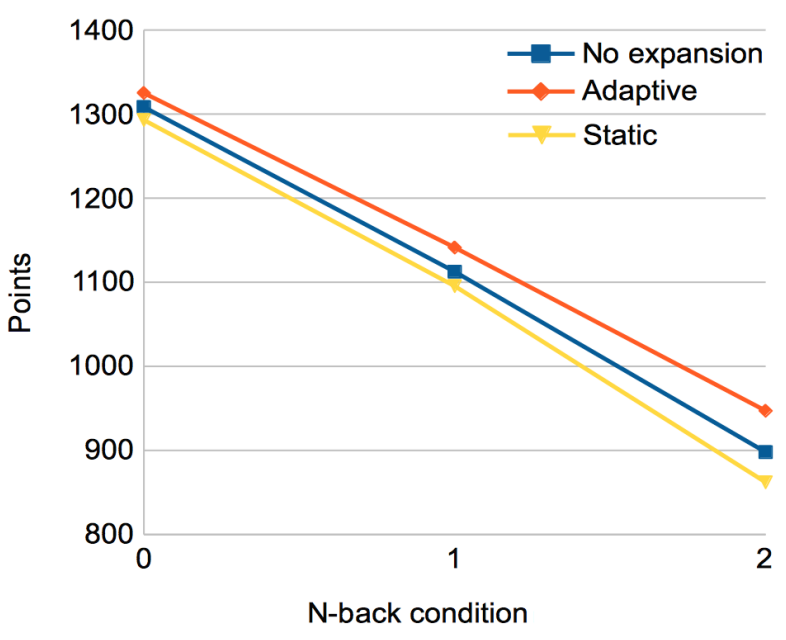

Figure 6. Points by expansion condition. Participants earned 20 points for each correct audio n-back response, while simultaneously earning ten points for each uppercase letter they clicked on and five points for each lowercase letter.

\section{Speed and Accuracy Results}

We found significant differences between conditions for average movement time across trials. Mauchly's test showed a violation of sphericity against Group $(W(2)=0.26, p<$ $0.01)$. We ran a one-way repeated-measure ANOVA and made Greenhouse-Geisser correction $(\varepsilon=0.57)$. It revealed a significant effect of target condition on movement time (in seconds), $F(2,10)=6.99, p<0.005$. A Tukey's pairwise comparison revealed significant differences between the adaptive and static conditions $(p<0.01)$ movement times as well as between the no expansion and static adaptation tasks $(p<0.1)$. We also used a two-way repeated-measure ANOVA and found a significant effect of $\mathrm{n}$-back condition $(p<.001)$ on movement time and a significant target expansion $\mathrm{X}$ n-back interaction on time $(p<.05)$, with all three $\mathrm{n}$-back conditions having significant differences $(p<.001)$.

A further analysis of movement time revealed that there was a significant difference across expansion $(F(2,10)=$ $2.24, p<.05)$ and n-back conditions $(F(2,10)=25.45, p<$ .001 ) in movement time for uppercase letters, as shown in Figure 5. The Tukey's pairwise comparison showed that adaptive expansion outperformed the no expansion $(p<.05)$ and static expansion conditions. In addition, movement time in the no expansion condition was significantly better than the static condition. There was no significant difference in expansion condition across lowercase letter movement times.

These results indicate that movement time actually becomes worse when we uniformly apply target expansion to the uppercase letters, but improves if we only do it when the user is in a state of branching.

Because bubble cursor ensures that a user always clicks on a target, we found the hit rate for all three conditions extremely high. There was no significant difference between expansion conditions in the 0-back and 1-back task, but we used a oneway ANOVA and found that in the 2-back, there was a signif- 


\begin{tabular}{|l|l|l|l|}
\hline Measure & No Expansion & Adaptive Expansion & Static Expansion \\
\hline N-back accuracy rate & $90.4 \%$ & $91.7 \%$ & $87.97 \%$ \\
\hline Number of targets selected (uppercase) & $116.5(66.9)$ & $122(67.7)$ & $114.58(66.2)$ \\
\hline Combined score (points) & 3358.75 & 3423.33 & 3278.75 \\
\hline Movement time - total (s) & 2.55 & 2.49 & 2.63 \\
\hline Movement time - uppercase (s) & 2.47 & 2.37 & 2.55 \\
\hline Movement time - lowercase (s) & 2.60 & 2.59 & 2.70 \\
\hline Target acquisition error rate & $1.63 \%$ & $1.52 \%$ & $2.80 \%$ \\
\hline
\end{tabular}

Table 1. Average results by expansion condition. All of these measures were significant $(p<.05)$ across expansion condition and $\mathbf{n}$-back condition except for movement time for lowercase targets and target acquisition error rate.

icant effect of condition $(F(2,10)=2.24, p<.05)$. Similar to Grossman and Balakrishnan, we choose not to do a full speed-accuracy analysis because our hit rates were well below the standard $4 \%$ error rate used to calculate target effective width in other target acquisition studies.

The index of difficulty across conditions was insignificant across target and n-back conditions, showing that participants performed roughly the same difficulty of task across conditions. We also looked at distance traveled, throughput (the ratio of index of difficulty to movement time), and path metrics, but did not find any differences across conditions. In addition, we found no learning effect either within trials of a condition or across conditions.

\section{DISCUSSION}

These results suggest that participants were able to perform more critical work with a higher accuracy rate in a brainbased target adaptive expansion system than in a standard bubble cursor interface. Our results show that we could create a continuous level of adaptation with only implicit, nonvisible assistance.

Although bubble cursor already shows large improvements over standard point cursors, we found additional performance increments using brain input to control target expansion for bubble cursor. Using expansion at the correct times helps users by an average of 60 milliseconds per click (100 milliseconds for high-priority targets). As expected, we find that performance increases when the user is selecting a highpriority target, but we are able to do this without a negative impact on low-priority targets. Although these slight increases are not dramatic, in a repeated task such as target acquisition, over time they can yield powerful changes in user capabilities. In addition, the user does slightly better in the primary audio task, which may indicate that the user is devoting less resources to the visual interface. Because of these gains, we see that the user is able to select an extra 5.5 targets on average. This means that the user is able to take advantage of the pointing performance increases and select more targets.

Although a calculated level of expansion helps the user, a constant maximum expansion actually impedes performance in both tasks. While we expected the static expansion condition to aid in acquisition of uppercase targets, we see that it only helps during low workload conditions and actually performs worst of all three conditions in the 2-back condition. We hypothesize this is due to the user spending extra cognitive and visual resources trying to figure out the limits of the target. In addition, although there is almost no difference between the other two conditions in selecting lowercase letters, the static expansion fares far worse in selecting lowercase letters.

From our results, it is clear that target adaptation alone is not enough to incite task improvements, but this adaptation must be judiciously applied, taking effect only when the user is dividing mental focus.

\section{ACKNOWLEDGEMENTS}

We thank Alvitta Ottley, Eli Brown, Fumeng Yang, Lane Harrison, Sergio Fantini, and Angelo Sassaroli from Tufts University, Erin Solovey from Drexel University, and R. Jordan Crouser from MIT Lincoln Laboratory. We also thank Google Inc. and the NSF for support of this research (NSF Grants Nos. IIS-1065154 and IIS-1218170). Any opinions, findings, conclusions, or recommendations expressed in this paper are those of the authors and do not necessarily reflect the views of Google Inc. or the National Science Foundation.

\section{REFERENCES}

1. Afergan, D., Peck, E. M., Solovey, E. T., Jenkins, A., Hincks, S. W., Brown, E. T., Chang, R., and Jacob, R. J. K. Dynamic Difficulty Using Brain Metrics of Workload. In Proc. ACM CHI 2014, ACM Press (2014).

2. Ayaz, H., Onaral, B., Izzetoglu, K., Shewokis, P. A., McKendrick, R., and Parasuraman, R. Continuous monitoring of brain dynamics with functional near infrared spectroscopy as a tool for neuroergonomic research: empirical examples and a technological development. Frontiers in Human Neuroscience 7 (2013).

3. Ayaz, H., and Willems, B. Cognitive workload assessment of air traffic controllers using optical brain imaging sensors. In Advances in Understanding Human Performance: Neuroergonomics, Human Factors Design, and Special Populations. CRC Press, 2010, 21-32.

4. Baddeley, A. Working memory. Science (1992).

5. Balakrishnan, R. Beating fitts' law: virtual enhancements for pointing facilitation. International Journal of Human-Computer Studies 61, 6 (2004), 857-874.

6. Baudisch, P., Zotov, A., Cutrell, E., and Hinckley, K. Starburst: a target expansion algorithm for non-uniform 
target distributions. Proceedings Working Conference on Advanced Visual Interfaces (2008).

7. Burgess, P. W., Veitch, E., de Lacy Costello, A., and Shallice, T. The cognitive and neuroanatomical correlates of multitasking. Neuropsychologia 38, 6 (2000), 848-63.

8. Chang, C., and Lin, C. LIBSVM: a library for support vector machines. ACM Transactions on Intelligent Systems and Technology (2011), 1-39.

9. Cockburn, A., and Gin, A. Faster cascading menu selections with enlarged activation areas. In Proc. Graphics interface 2006, Canadian Information Processing Society (2006).

10. Cui, X., Bray, S., and Reiss, A. L. Speeded near infrared spectroscopy (NIRS) response detection. PLoS one 5, 11 (2010).

11. Curtis, C. E., and D'Esposito, M. Persistent activity in the prefrontal cortex during working memory. Trends in Cognitive Sciences 7, 9 (2003), 415-423.

12. Cutrell, E., and Tan, D. BCI for passive input in HCI. In Proc. CHI 2008, ACM Press (2008).

13. D'Esposito, M., Zarahn, E., and Aguirre, G. K. Event-related functional MRI: implications for cognitive psychology. Psychological Bulletin 125, 1 (1999), 155.

14. Fabiani, G., and McFarland, D. Conversion of EEG activity into cursor movement by a brain-computer interface (BCI). IEEE Transactions on Neural Systems and Rehabilitation Engineering 12, 3 (2004).

15. Fairclough, S. H. Fundamentals of physiological computing. Interacting with Computers 21, 1-2 (2009), $133-145$.

16. Girouard, A., and Solovey, E. Distinguishing Difficulty Levels with Non-invasive Brain Measurements. INTERACT 2009, Figure 1 (2009), 440-452.

17. Girouard, A., Solovey, E. T., and Jacob, R. J. K. Designing a passive brain computer interface using real time classification of functional nearinfrared spectroscopy. International Journal of Autonomous and Adaptive Communications Systems 6, 1 (2013), 26-44.

18. Gray, W. D., and Boehm-Davis, D. A. Milliseconds matter: An introduction to microstrategies and to their use in describing and predicting interactive behavior. Journal of Experimental Psychology: Applied 6, 4 (2000), 322.

19. Grossman, T., and Balakrishnan, R. The bubble cursor: enhancing target acquisition by dynamic resizing of the cursor's activation area. In Proc. CHI 2005, ACM Press (2005).

20. Guiard, Y., Blanch, R., and Beaudouin-Lafon, M. Object pointing: a complement to bitmap pointing in GUIs. In Proc. Graphics Interface 2004 (2004), 9-16.
21. Guiard, Y., Olafsdottir, H. B., and Perrault, S. T. Fitts' Law as an Explicit Time/Error Trade-Off. In Proc. CHI 2011, ACM Press (2011).

22. Han, S.-H., and Kim, M.-S. Visual search does not remain efficient when executive working memory is working. Psychological Science 15, 9 (2004), 623-8.

23. Harrison, J., Izzetoglu, K., and Ayaz, H. Human Performance Assessment Study in Aviation Using Functional Near Infrared Spectroscopy. Foundations of Augmented Cognition (2013), 433-442.

24. Hirshfield, L., Gulotta, R., and Hirshfield, S. This is your brain on interfaces: enhancing usability testing with functional near-infrared spectroscopy. In Proc. CHI 2011, ACM Press (2011).

25. Hirshfield, L. M., Solovey, E. T., Girouard, A., Kebinger, J., Jacob, R. J. K., Sassaroli, A., and Fantini, S. Brain Measurement for Usability Testing and Adaptive Interfaces: An Example of Uncovering Syntactic Workload with Functional Near Infrared Spectroscopy. In Proc. CHI 2009, ACM Press (2009).

26. Huppert, T., and Hoge, R. A temporal comparison of BOLD, ASL, and NIRS hemodynamic responses to motor stimuli in adult humans. Neuroimage 29, 2 (2006), 368-382.

27. Kabbash, P., and Buxton, W. The "Prince" Technique : Fitts' Law and Selection Using Area Cursors. In Proc. CHI 1995, ACM Press (1995).

28. Kelso, J. Theoretical concepts and strategies for understanding perceptual-motor skill: From information capacity in closed systems to self-organization in open, nonequilibrium. Journal of Experimental Psychology: General (1992).

29. Koechlin, E., Basso, G., Pietrini, P., Panzer, S., and Grafman, J. The role of the anterior prefrontal cortex in human cognition. Nature 399, 6732 (1999), 148-51.

30. MacKenzie, I. S. Fitts' law as a research and design tool in human-computer interaction. Human-computer interaction (1992).

31. Maior, H. A., and Pike, M. Continuous Detection of Workload Overload: An fNIRS Approach. In Proc. International Conference on Ergonomics \& Human Factors 2014, CRC Press (2014).

32. McGuffin, M., and Balakrishnan, R. Fitts' law and expanding targets: Experimental studies and designs for user interfaces. Transactions on Computer-Human Interaction 12, 4 (2005), 388-422.

33. McKendrick, R., Ayaz, H., Olmstead, R., and Parasuraman, R. Enhancing dual-task performance with verbal and spatial working memory training: continuous monitoring of cerebral hemodynamics with NIRS. Neuroimage 85 (2014), 1014-1026. 
34. Mehler, B., Reimer, B., and Coughlin, J. F. Sensitivity of Physiological Measures for Detecting Systematic Variations in Cognitive Demand From a Working Memory Task: An On-Road Study Across Three Age Groups. Human Factors: The Journal of the Human Factors and Ergonomics Society 54, 3 (2012), 396-412.

35. Mehler, B., Reimer, B., Coughlin, J. F., and Dusek, J. A. Impact of Incremental Increases in Cognitive Workload on Physiological Arousal and Performance in Young Adult Drivers. Transportation Research Record: Journal of the Transportation Research Board 2138, 2 (2009), 6-12.

36. Mott, M. E., and Wobbrock, J. O. Beating the bubble: using kinematic triggering in the bubble lens for acquiring small, dense targets. In Proc. CHI 2014, ACM Press (2014).

37. Peck, E. M., Afergan, D., and Jacob, R. J. K. Investigation of fNIRS brain sensing as input to information filtering systems. In Proc. Augmented Human International, ACM Press (2013).

38. Peck, E. M., Afergan, D., Yuksel, B. F., Lalooses, F., and Jacob, R. J. Using fNIRS to measure mental workload in the real world. In Advances in Physiological Computing. Springer, 2014, 117-139.

39. Pike, M. F., Maior, H. A., Porcheron, M., Sharples, S. C., and Wilson, M. L. Measuring the effect of think aloud protocols on workload using fNIRS. In Proc. CHI 2014, ACM Press (2014).

40. Reimer, B., Mehler, B., Coughlin, J. F., Godfrey, K. M., and Tan, C. An on-road assessment of the impact of cognitive workload on physiological arousal in young adult drivers. Proc. AutomotiveUI 2009 (2009).

41. Repovš, G., and Baddeley, A. The multi-component model of working memory: explorations in experimental cognitive psychology. Neuroscience 139, 1 (2006), 5-21.

42. Sassaroli, A., Zheng, F., Hirshfield, L. M., Girouard, A., Solovey, E. T., Jacob, R. J. K., and Fantini, S. Discrimination of Mental Workload Levels in Human Subjects with Functional Near-Infrared Spectroscopy. Journal of Innovative Optical Health Sciences 1, 2 (2008), 227-237.
43. Schalk, G., McFarland, D. J., Hinterberger, T., Birbaumer, N., and Wolpaw, J. R. BCI2000: a general-purpose brain-computer interface (BCI) system. IEEE Transactions on Biomedical Engineering 51, 6 (2004), 1034.

44. Solovey, E. T., Girouard, A., Chauncey, K., Hirshfield, L. M., Sassaroli, A., Zheng, F., Fantini, S., and Jacob, R. J. K. Using fNIRS Brain Sensing in Realistic HCI Settings: Experiments and Guidelines. In Proc. UIST 2009, ACM Press (2009).

45. Solovey, E. T., Girouard, A., Jacob, R. J., Lalooses, F., Chauncey, K., Weaver, D., Parasi, M., Scheutz, M., Sassaroli, A., Fantini, S., and Schermerhorn, P. Sensing cognitive multitasking for a brain-based adaptive user interface. Proc. CHI 2011 (2011).

46. Solovey, E. T., Schermerhorn, P., Scheutz, M., Sassaroli, A., Fantini, S., and Jacob, R. Brainput: Enhancing Interactive Systems with Streaming fNIRS Brain Input. In Proc. CHI 2012, ACM Press (2012).

47. Vidaurre, C., Kawanabe, M., von Bünau, P., Blankertz, B., and Müller, K. R. Toward unsupervised adaptation of LDA for brain-computer interfaces. IEEE Transactions on Biomedical Engineering 58, 3 (2011), 587-97.

48. Villringer, A., and Chance, B. Non-invasive optical spectroscopy and imaging of human brain function. Trends in neurosciences 20, 10 (1997), 435-42.

49. Wickens, C. Multiple resources and mental workload. Human Factors: The Journal of the Human Factors and Ergonomics Society 50, 3 (2008), 449-455.

50. Worden, A., Walker, N., Bharat, K., and Hudson, S. Making computers easier for older adults to use: area cursors and sticky icons. In Proc. CHI 1997, ACM Press (1997).

51. Zander, T. O., Kothe, C., Welke, S., and Rötting, M. Utilizing secondary input from passive brain-computer interfaces for enhancing human-machine interaction. In Foundations of Augmented Cognition, Neuroergonomics and Operational Neuroscience, Springer Berlin Heidelberg (2009), 759-771.

52. Zhai, S., and Conversy, S. Human on-line response to target expansion. In Proc. CHI 2003, ACM Press (2003). 